Stoa

Vol. 3, No. 5, 2012, pp. 65-85

ISSN 2007-1868

\title{
LEYES FUNDAMENTALES, A PRIORI RELATIVIZADOS Y GÉNEROS*
}

\author{
José Luis Falguera \\ Facultad de Filosofía \\ Universidad de Santiago \\ de Compostela \\ joseluis.falguera@usc.es
}

RESUMEN: La que se conoce como "teoría de la estructura léxica o taxonómica” tiene especial relevancia en los últimos artículos de Kuhn y en relación con ella el papel de "los términos/conceptos de género" también es significativo. En este trabajo pretendo establecer que la teoría de adquisición/aprendizaje de estos términos considerada por Kuhn es una suerte de holismo epistémicosemántico en relación a ciertas generalizaciones con rol estipulativo o generalizaciones que expresan proposiciones “a priori relativizadas". Defiendo que los análisis de Moulines sobre la forma lógica relevante de las leyes fundamentales (o principios guía) proporcionan un claro apoyo a la idea de que una ley fundamental es una "proposición a priori relativizada". También se defiende que la concepción estructuralista -según el enfoque de Moulines- proporciona cierto apoyo al carácter constitutivo de los términos de género.

PALABRAS CLAVE: términos/conceptos de género $\cdot$ leyes fundamentales $\cdot$ a priori relativizados $\cdot$ Kuhn $\cdot$ metateoría estrcuturalista

ABST RACT: The so-called "theory of lexical or taxonomic structure" has special weight in Kuhn's latest articles and, as a result, the role of "kinds terms/concepts" is also significant. In this work, I intend to establish that the theory of acquisition/learning for those terms/concepts considered by Kuhn is a sort of epistemic-semantic holism concerning some generalizations with a stipulative role or generalizations which express propositions "relativized a priori". I defend that Moulines' analyses about the relevant logical form of fundamental laws (or guide principles) give a clear support to the idea that a fundamental

\footnotetext{
* Este trabajo se elaboró en el marco de los proyectos de investigación: HUM200604955/FISO y FFI2009-08828/FISO del Ministerio de Educación y Ciencia de España; así como PICTR2002 Nº0219 y PICTR2006 № 2007 de la Agencia Nacional de Promoción Científica y Tecnológica de Argentina. Dicho trabajo se ha visto enriquecido gracias a los comentarios de J. A. Díez, J. M. Jaramillo, C. Lorenzano y P. Lorenzano. Ninguno de ellos es responsable de las deficiencias que pueda encerrar. A todos ellos mi agradecimiento.
} 
law is a "relativized a priori proposition". I also advocate that the structuralist view - according to the Moulines approach - about fundamental laws provides some support to the constitutive character of kinds concepts.

KEYWORDS: kind terms/concepts · fundamental laws · relativized a priori $\cdot$ Kuhn - metateoría estructuralista

\section{Introducción}

Desde sus orígenes, con el texto de Sneed de 1971, la metateoría estructuralista ha tenido presentes las aportaciones de Kuhn acerca del desarrollo de las teorías científicas y de los elementos relevantes en la comprensión de tal desarrollo, contribuyendo de igual forma a una interpretación clarificadora de los planteamientos de éste. Cabe decir que desde 1976, año en que Kuhn publica un comentario crítico de los planteamientos de Sneed y de sus desarrollos posteriores realizados por Stegmüller (en especial los de 1973), los puntos de vista de la metateoría estructuralista también influyeron en su filosofía.

Especial relevancia tiene en sus últimos artículos lo que se conoce como "la teoría de la estructura léxica o taxonómica" y en relación con ella el papel de "los géneros" y de "los términos/conceptos (de género)" mediante los que se designa/denota a los primeros. Uno de los propósitos de este trabajo es mostrar que el bagaje sobre el que Kuhn da cuenta de los términos/conceptos de género es el de la metateoría estructuralista; en especial se pretende establecer que la teoría de adquisición/aprendizaje de tales términos/conceptos es manejada por él como una suerte de holismo epistémico-semántico en relación a ciertas generalizaciones con rol estipulativo. No es difícil ver tras esas generalizaciones a las leyes fundamentales, características en la metateoría estructuralista de lo que se presenta como un elemento teórico básico (de una red teórica arbórea) pero, además, cada generalización con éste rol es presentada como un "a priori relativizado" (adoptando esta noción de Reichenbach, tal y como ha sido recientemente propuesto por Friedman).

En este trabajo se defenderá que el análisis de Moulines acerca de la forma lógica relevante de las leyes fundamentales (o principios guía) proporciona un claro apoyo a la idea de que una ley fundamental es una proposición a priori relativizada. La finalidad es intentar defender que la consideración estructuralista -more Moulines- acerca de las le- 
yes fundamentales da lugar a apoyar el carácter constitutivista (aunque no constructivista) de los géneros, lo cual está en consonancia con los últimos puntos de vista de Kuhn.

\section{Adentrándose escuetamente en el panorama actual de la concepción estructuralista}

Dicen Díez y Lorenzano en la contextualización que hacen de la concepción estructuralista en el volumen titulado Desarrollos actuales de la metateoría estructuralista: problemas y discusiones, el cual recoge los trabajos presentados con motivo de un encuentro acerca de la concepción estructuralista celebrado en Zacatecas, que en el período contemporáneo (que se inicia en la década de los 70) una corriente de filosofía de la ciencia

muestra [ni más ni menos] tras el repliegue de los primeros efectos antiformalistas, que al menos parte de los nuevos elementos señalados durante el período historicista son susceptibles de un razonable análisis y reconstrucción formal. (Díez y Lorenzano 2002, p. 27)

Estrictamente bajo tal corriente, que genéricamente se conoce como corriente semántica o teórico-modelista, se integra una familia de concepciones entre las que destacan las aportaciones de la metateoría de espacios de estado, que tiene como representantes destacados a B. Van Fraassen y a F. Suppe, y la de la metateoría estructuralista, representada por J. Sneed -su iniciador-, W. Stegmüller, C. U. Moulines y W. Balzer, ${ }^{1}$ mi interés se centra en esta última concepción.

Hoy en día cabe decir que la metateoría estructuralista ha hecho significativas aportaciones a la filosofía de la ciencia, entre las que destacan las que conciernen a: la elucidación sincrónica y diacrónica (y en este último caso especialmente a los aspectos cinemáticos) de las teorías empíricas y con múltiples aplicaciones en la reconstrucciones de teorías concretas; la distinción entre lo teórico/no-teórico de los conceptos característicos de todos los principios (en tanto que relativizada a cada uno de ellos ), a la luz de un criterio preciso de teorici$\mathrm{dad} ;{ }^{2}$ el análisis de la aserción empírica de una teoría a la luz del de-

\footnotetext{
${ }^{1}$ La corriente semánticista incluye precedentes importantes como los debidos a J.J.C. McKinsey, E. Beth, J. von Neumann, G. Birkhoff, P. Suppes y E.W. Adams.

${ }^{2}$ Estrictamente un par de criterios diferentes: uno que incorpora elementos pragmáticos (y que fue el que inicialmente se buscó, siguiendo originalmente una distinción debida a
} 
nominado "enunciado Ramsey-Sneed"; la elucidación de nociones de relaciones intra e interteóricas, como la de especialización, la de teorización, las de reducción y las de equivalencia (empírica y completa); la elucidación de nociones de aproximación para una consideración más apropiada de los productos cognoscitivos de las ciencias empíricas; así como la incorporación de distinciones conceptuales útiles en el análisis (cuasi-formal) de las teorías empíricas y sus componentes.

Pero también es cierto que hay aspectos de esta concepción que han sido abordados en la literatura sin que hayan obtenido hasta ahora un tratamiento mayoritariamente aceptado entre los conocedores de la corriente. ${ }^{3}$ Sin hacer referencia al tópico realismo vs. anti-realismo, que inevitablemente resurge a la luz de todo enfoque de filosofía de la ciencia, encontramos que ya Moulines recordaba en un trabajo inicialmente presentado en el congreso en Zacatecas, mencionado líneas arriba, que tras los análisis formales de la metateoría estructuralista en la representación de las teorías se agazapa la pragmática ${ }^{4}$, con los problemas abiertos que ello supone para la comprensión de las propias teorías. Por otro lado, en muchos debates y trabajos del ámbito de esta concepción los problemas ontosemánticos de las expresiones científicas han ido abriéndose paso sin que pueda decirse, a mi entender y dadas las diferentes estrategias adoptadas para abordarlos, que esté claro el panorama. ${ }^{5}$ Los problemas del holismo epistémico y del holismo semántico, por más que han sido objeto de diferentes consideraciones, parecen eludir un tratamiento satisfactorio. Pero además de estos problemas, y otros que se me escapan, hay dos que son centrales para la comprensión de los productos cognoscitivos de la ciencia

Hempel) y otro de carácter formal. (Para ambos véase Balzer, Moulines y Sneed 1987, pp. 47-73 y 391-393 para el pragmático y pp. 73-78 para el formal).

${ }^{3}$ No estoy pretendiendo que la concepción estructuralista dé cuenta de los problemas de metateoría de la ciencia que existan (ni siquiera de aquellos que se suelen asociar a la filosofía de la ciencia), como a veces los detractores de la concepción injustamente reclaman. Como todo planteamiento serio, la metateoría estructuralista no es abarcadora del campo de la metaciencia, ni siquiera del de la filosofía de la ciencia; sólo se ha ido ocupando de algunos problemas, especialmente el de la identidad y caracterización cuasi-formal (con rigor y cierta precisión) de las teorías empíricas. Esto es de interés en la medida en que sus aportaciones se consideren adecuadas al permitir analizar con rigor y cierta precisión otros problemas metateóricos.

${ }^{4}$ Cfr. Moulines 2002.

${ }^{5}$ Moulines, Balzer, Lauth, Zoubek, Díez y yo mismo, al menos, hemos hecho contribuciones a este asunto. (Cfr. texto y referencias en Falguera 2002). 
y que resurgen al tomarse en serio los aspectos epistémicos y los ontosemánticos (o si se prefiere, los ontoepistemosemánticos, como nos quiere acostumbrar a decir Moulines) y que en definitiva constituyen problemas que afectan a la propia comprensión de las teorías, especialmente cuando pasamos de la noción básica de teoría (o "elemento teórico") a una más rica y compleja (ya sea meramente estática, como la de "árbol teórico", o cinemática, como la de "evolución teórica”). Me refiero, por un lado, al problema de la base empírica de cada teoría y, por el otro, al del estatus de las "leyes fundamentales" (es decir, el de la ley o las leyes asociadas al elemento teórico básico [basic theory-element] de un árbol teórico [theory-tree]) completo).

Aquí voy a ocuparme del último problema. En otros lugares he intentado decir algo sobre la base empírica y aquí sólo señalaré, a fin de ser un poco provocativo sobre este asunto, que considero errada la pretensión de perseguir un fundamento último para toda teoría empírica en modelos de datos que conecten finalmente nuestras teorías y sus conceptos característicos con una base perceptiva, pero esto no significa que no haya base empírica (o evidencia, como yo prefiero llamarla) para una teoría o modelo de datos, sino que no necesariamente ha de estar basada en información de carácter perceptivo.

El problema del estatus de las leyes fundamentales, junto con el problema de la base empírica o evidencia, nos remontan a los clásicos problemas epistémicos del racionalismo vs. el empirismo aunque, haciendo una concesión más a Moulines, en realidad creo que nos ubican en los dos extremos relevantes de la ontoepistemosemántica de la ciencia. En lo restante pretendo explorar el asunto de las leyes fundamentales por los siguientes motivos:

- En primer lugar para mostrar que ya hay aportaciones significativas al respecto en la literatura, y en este sentido me ocuparé inicialmente de los desarrollos últimos de Kuhn y del análisis que Moulines hizo de las leyes fundamentales (o principios guía') en $1978 .{ }^{6}$

${ }^{6}$ Cfr. Moulines 1978. Incluido con ligeras modificaciones en Moulines 1982, cap. 2.3. 
- En segundo lugar para reconsiderar estas propuestas a la luz de la noción de a priori relativizado, acuñada (hasta donde yo sé) por Reichenbach. ${ }^{7}$

- En tercer lugar para extraer algunas consecuencias ontoepistemosemánticas de tales consideraciones, que están en la línea de las asumidas por Kuhn al tomar géneros como referencia de los términos científicos.

- En cuarto lugar para elucidar un criterio de ley fundamental que proviene de análisis desarrollados en la metateoría estructuralista.

\section{Kuhn, la metateoría estructuralista y las leyes fundamentales}

Es habitual desde los inicios de la concepción estructuralista, con la obra de Sneed (1971), diferenciar en una teoría empírica compleja entre su núcleo teórico central (o estructural) y expansiones del mismo alcanzadas por vía de especialización. El núcleo teórico tiene como elemento identificativo principal una o varias leyes centrales y las expansiones se identifican básicamente por las sucesivas leyes especiales que se anexionan, con aplicación más restringida (por lo general) a aquellas leyes centrales. ${ }^{9}$ A partir de la publicación de Theorienstrukturen und Theoriendynamik [Estructura y dinámica de teorías] por parte de Stegmüller en $1973,{ }^{10}$ las leyes centrales de una teoría pasan a ser conocidas en la literatura estructuralista como leyes fundamentales, y el núcleo estructural central se conoce en las presentaciones actuales de

${ }^{7}$ Cfr. Reichenbach 1920/1965, cap. V. Jaramillo, en una conferencia titulada "Tópicos kantianos en la concepción estructuralista", ${ }^{8}$ apela también a un a priori relativizado, presente, según él, en algunos de los epígonos de Kant, como apropiado para dar cuenta de las leyes fundamentales de la metateoría estructuralista; ésta conferencia fue impartida en Xalapa, Veracruz-México, en julio de 2004, con motivo del congreso "30 Años de Estructuralismo: Resultados y Perspectivas/30 Years of Structuralism: Results and Perspectives", en el que también yo presenté una versión de este trabajo.

${ }^{9}$ Prescindo, por mor de la simplicidad, de mencionar otros componentes identificados por la metateoría estructuralista.

${ }^{10}$ Cfr. Stegmüller 1973 [v.c., 1983, p. 142, pp. 161 y ss.]. Estrictamente, Stegmüller habla en ese texto de ley fundamental para referirse a la totalidad del predicado conjuntista que tiene como extensión los modelos actuales de una teoría. Con posterioridad la noción de ley fundamental se utiliza para dar cuenta del axioma o axiomas propios -una(s) fórmula(s) o enunciación(es), pues- que forma o forman parte de la definición de aquel predicado conjuntista que tiene como extensión los modelos actuales del elemento teórico básico de una teoría compleja (de un árbol teórico). 
la metateoría estructuralista como núcleo teórico del elemento teórico básico de una teoría compleja madura (de un árbol teórico). Sin embargo, las menciones explícitas a las leyes fundamentales de las teorías no son del todo frecuentes en la literatura de la metateoría estructuralista y menores son las consideraciones acerca de su estatus. No deja de ser sintomático que en la obra magna de esta concepción, a saber, An Architectonic for Science (de Balzer, Moulines y Sneed) sólo se mencionan de pasada (sin más especificaciones) al comienzo del texto. ${ }^{11}$ Claro que en dicha literatura se habla del elemento estructural al que está asociado una ley fundamental -i.e. se habla del núcleo teórico básico como parte esencial de una teoría compleja y madura que es "muy poco o nada restrictiva", ${ }^{12}$ y se habla del elemento teórico del que forma parte tal partícula estructural denominándolo elemento teórico fundamental' (o básico) y señalando que "es de naturaleza conceptual completamente general". ${ }^{13}$

Las excepciones a tal ausencia de referencias las proporcionan Stegmüller y Moulines. El primero llama la atención en diferentes textos acerca de la vacuidad de las leyes fundamentales de las teorías y del papel nuclear de una ley fundamental en el desarrollo normal de cada una de éstas. Con ello, trata de explicar las ideas de corte kuhniano (y lakatosiano) de (i) la inmunidad de una teoría a la falsación, dada tal vaciedad de una ley fundamental, y (ii) la importancia de preservar la(s) ley(es) fundamental(es) de una teoría aunque varíen las especiales y algunos otros elementos de ésta para poder decir que estamos ante la misma teoría (lo que Kuhn denominaría desarrollo de la ciencia normal'). ${ }^{14}$

Conforme al planteamiento de Stegmüller tenemos que los cambios revolucionarios de teoría a la Kuhn, es decir, los desplazamientos de una teoría por otra alternativa que conllevan inconmensurabilidad, suponen cambios de la(s) ley(es) fundamental(es) suplantada(s) por una(s) ley(es) fundamental(es) nueva(s) alternativa(s) característica(s) de la nueva teoría. Desde este punto de vista la vaciedad de leyes y, a pesar de ello, la importancia de su preservación para la identi-

${ }^{11} \mathrm{Cfr}$. Balzer, Moulines y Sneed 1987, p. 15.

${ }^{12}$ Cfr. Díez y Moulines 1997, p. 363.

${ }^{13}$ Cfr. Balzer-Moulines-Sneed 1987, p. 175.

${ }^{14}$ Cfr. Stegmüller 1976 , pp. 162 y ss.; 1978 , p. $168 ; 1979$, pp. $74-75$. 
dad de una teoría, son caras de la misma moneda, peculiaridades que conjuntamente requieren ser explicadas.

Moulines también hace uso de la noción de ley fundamental, muy especialmente en la parte III de Pluralidad y recursión, diferenciando las normas fundamentales de las especiales. Tras establecer que las primeras "contienen la «información esencial»", Moulines indica que:

si bien no hay unanimidad en algún criterio metodológico general para considerar un enunciado cualquiera como una ley, en cambio, en cada caso particular de reconstrucción de una teoría dada, parece, por regla general, ser relativamente fácil concordar, en base a consideraciones informales o semiformales (por ejemplo, sobre su papel sistematizador o su carácter cuasi-vacuo), en que un determinado enunciado debe tomarse como ley fundamental de la teoría en cuestión. [...] si bien no podemos indicar condiciones necesarias y suficientes para determinar que un enunciado es una ley fundamental, es posible en cambio señalar ciertos 'síntomas", algunos incluso formalizables.

Y entre los síntomas, además del carácter sistematizador y/o cuasivacuo (empíricamente), señala el 'carácter sinóptico' de las leyes fundamentales, añadiendo que:

Con carácter sinóptico de una ley fundamental de una teoría me refiero al hecho de que cualquier formulación correcta de la ley deberá incluir necesariamente todos los conceptos fundamentales que caracterizan dicha teoría. (Moulines 1991, pp. 233-234)

A pesar de esto, reconoce que no todos los candidatos plausibles a leyes fundamentales revelan poseer ese rasgo sinóptico; ${ }^{15}$ no obstante, si bien no toda ley fundamental debe satisfacer dicho "carácter sinóptico", al menos cabe pensar que el conjunto de leyes fundamentales de una teoría madura deben proporcionar todos los conceptos característicos de la misma, y aunque haya casos en los que cabe diferenciar varias leyes fundamentales, cada una de éstas no se aplica aisladamente. Por lo demás, debe resultar obvio que el supuesto carácter cuasi-vacuo de las leyes fundamentales equivale a que éstas apenas restringen sobre el ámbito de modelos potenciales, mientras que las leyes especiales sí son totalmente restrictivas.

${ }^{15} \mathrm{Al}$ respecto indica como ejemplos las leyes fundamentales de la mecánica relativista del continuo y de la electrodinámica, a la luz de la reconstrucción de Bartelborth (Cfr. Bartelborth 1988, cap. I). 
Hay otro texto previo de Moulines en el que habla de lo que hemos venido presentando hasta aquí como leyes fundamentales, en el que las denomina "principios guía". Se trata de un texto inicialmente publicado en 1978 y finalmente recogido como capítulo de Exploraciones metacientíficas con el título de "Forma y función de los principios-guía en las teorías físicas". Los ejemplos que maneja en este texto son sólo de dos teorías de la física, a saber, la mecánica clásica y la termodinámica fenomenológica, aunque quizás algunos de los rasgos característicos sean extensibles a casos de leyes fundamentales de otras teorías. Más adelante volveré sobre este texto, de momento sólo quiero apuntar que Moulines asocia las propuestas acerca de los principios guías a los "paradigmas" de Kuhn (en alguna de las acepciones de esta expresión en su texto de 1962).

Sin duda tal consideración es una muestra más del influjo que la obra de Kuhn ha tenido en las aportaciones de estructuralistas destacados y, por ello, en la concepción estructuralista; sin embargo, desde mi punto de vista apenas ha sido considerada la influencia de esta concepción en la obra de Kuhn.

Thomas Kuhn publica en 1976 un texto pocas veces mencionado en las bibliografías por sus comentaristas y aun cuando aquellas lo recogen, apenas es considerado en los trabajos de los que éstas forman parte, se trata de su trabajo "Theory-Change as Structure-Change: Comments on the Sneed Formalism" ["El cambio de teoría como cambio de estructura: comentarios sobre el formalismo de Sneed"], con el mismo, a mi entender, comienza una nueva fase en su reflexión en el ámbito de la filosofía de la ciencia, en la que introducen importantes variantes con respecto a su obra previa. En dicho texto Kuhn nos da a conocer sus incursiones en la literatura estructuralista de los primeros momentos y por más que manifiesta algunas diferencias con consideraciones de Sneed y Stegmüller - muy especialmente con la posibilidad de que ante casos de revolución científica la vieja teoría se reduzca (en un sentido no deductivo) ${ }^{16}$ a (un caso especial de) la nueva-, deja bien claro que considera que el enfoque formal de estos autores (y por tanto de la llamada concepción estructuralista) es el enfoque filosófico más próximo a sus planteamientos desarrollados hasta ese momento. Además,

\footnotetext{
${ }^{16}$ Conviene aclarar que la reducción estructuralista no precisa ser estrictamente deductiva, a diferencia de la reducción interteórica del neopositivismo.
} 
ese trabajo sirve a Kuhn para reafirmar su tesis de la inconmensurabilidad interteórica, aunque presentada (como ya venía haciendo en textos previos) como inconmensurabilidad local y, sobre todo, como restringida al aspecto lingüístico-conceptual, es decir, a la imposibilidad de traducción a un lenguaje común de ciertos pares de teorías, con el fin de que ésta permita su comparación punto por punto (aunque sea posible cotejarla por otras vías que no sean de carácter lógicodeductivo). Con ello, Kuhn se olvida de otros aspectos que habían formado parte de sus consideraciones previas en la caracterización de la inconmensurabilidad, como son los métodos, normas y valores, o el aspecto perceptivo.

Pero es determinante de este planteamiento el que vincule la tesis de la inconmensurabilidad con una tesis de holismo semántico local. Si resalto lo de local es porque previamente Kuhn ya había vinculado la tesis de la inconmensurabilidad con una tesis semántica holista, pero se trataba de la tesis global de orientación quineana. A partir del texto de 1976 Kuhn se distancia de los puntos de vista quineanos para abrazar un holismo semántico de menor alcance. Conforme al mismo, sólo ciertos términos en cada teoría están intrínsecamente conectados, de manera tal que sus significados son interdependientes. ${ }^{17}$ Los cambios de significado en tales casos se producen conjuntamente en un grupo de términos y afectan a la manera en que se categoriza la naturaleza; es decir, a lo que son los tipos de entidades consideradas y a qué se toma como objetos o situaciones cayendo bajo cada tipo considerado. ${ }^{18}$ Las revoluciones científicas se caracterizan por cambios de esa índole, que Kuhn empieza a denominar "cambios en varias de las categorías taxonómicas" y que suponen modificaciones respecto a las relaciones de semejanza que conforman tales categorías en la teoría desplazada. ${ }^{19}$

Lo que Kuhn denomina el significado de un término no debe confundirse con el conjunto de modos de determinación o criterio: él está lejos de adoptar el enfoque que identifica el significado de una expresión con el conjunto de modos de determinación de las instancias que conforman su extensión total -o con algún subconjunto suficientemente amplio. En concreto, no puede considerar el significado de

${ }^{17}$ Cfr. Kuhn 1983, p. 130 [v.c. p. 682]; 1987, p. 55 [v.c. p. 71].

${ }^{18}$ Cfr. Kuhn 1983, p. 131 y ss. [v.c. pp. 682 y ss.]; 1987, p. 88 [v.c. p. 19].

${ }^{19}$ Cfr. Kuhn 1987, p. 88 y ss. [v.c. p. 19 y ss.]. 
cada término científico como dado por los modos de determinación o criterios con los que ese término se relaciona con la naturaleza, porque, según él, los criterios para un término cambian con el desarrollo de su teoría, sin que ello conlleve cambio de bases, ni por lo tanto del significado del término ${ }^{20} \mathrm{y}$, es más, diferentes usuarios de una misma teoría pueden disponer de criterios diferentes para un determinado término de ella y no por ello se podría pensar que manejan nociones -significados- diferentes. ${ }^{21}$ En realidad Kuhn parece restringir el significado de un término científico a la categoría que éste conforma, en interdependencia con otros términos de una teoría, para estructurar -configurar, constituir- la naturaleza. Los términos de una teoría que son interdependientes constituyen lo que él llama una estructura léxica o estructura taxonómica. ${ }^{22}$ Diferentes modos de determinación pueden ser asociados a un término sin que ello suponga cambio de la categoría que conforma.

No deja de ser clarificador que desde 1976 este tipo de consideraciones, en especial las que tienen que ver con la interdependencia de ciertos términos en una teoría, se hagan por parte de Kuhn mencionando a los análisis de Sneed y Stegmüller. Mi punto de vista es que la tesis del holismo semántico local es resultado de la influencia de los planteamientos estructuralistas y en especial de su manera de considerar la distinción teórico/no-teórico y el papel que en relación con tal distinción juegan las leyes fundamentales de cada teoría; si esto es cierto, cabe decir que la concepción estructuralista, que evidentemente ha bebido de las fuentes del Kuhn inicial, a su vez ha condicionado los análisis de éste sobre filosofía de la ciencia en sus últimos años.

Pero cabe decir que además, sobre la base de la tesis del holismo semántico local, Kuhn desarrolla ideas originales en la línea de lo que antes presenté como "la teoría de la estructura taxonómica o del léxico". En concreto, la estructura taxonómica o el léxico de una teoría se asocia a ciertos términos que él llama indistintamente "términos taxonómicos" o "términos de género" [kind terms]. La noción de "género" $[k i n d]$ pasa a ser central en sus últimos trabajos; no obstante, debe quedar claro que, aunque en los primeros textos en que habla de géneros

${ }^{20}$ Cfr. Kuhn 1987, pp. 87 y ss. y nota 13 [v.c. pp. 19 y ss. y nota 13].

${ }^{21}$ Cfr. Kuhn 1983, pp. 127 y ss. [v.c. pp. 681 y ss.].

${ }^{22}$ Cfr. Kuhn 1983, pp. 131-132 [v.c. pp. 682-683]. 
utiliza la expresión "géneros naturales", su mención de los géneros no tiene connotaciones realistas como las que a veces se manejan cuando se habla de géneros naturales y, en todo caso, en sus últimas publicaciones pasa a hablar simplemente de géneros, dejando claro que éstos abarcan un abanico amplio en el que se incluyen los géneros de artefactos, los géneros sociales, etc. ${ }^{23}$ Los conceptos expresados mediante términos de género científicos fijan maneras de recortar la naturaleza maneras de cómo constituirla conforme a determinada teoría en la que se usen tales conceptos.

$\mathrm{Al}$ respecto encontramos que Kuhn hace diversas indicaciones que apuntan en ese sentido:

(1) los conceptos de género están agrupados conformando una determinada estructura léxica (un esquema conceptual), "un modo particular de operar de un módulo mental que es prerrequisito para tener creencias, un modo que a la vez provee y limita el conjunto de creencias que es posible concebir"; ${ }^{24}$

(2) los términos de género específicos de una teoría requieren de un vocabulario previamente disponible [antecedent vocabulary]; 25

(3) los términos de género que conforman un léxico para la ciencia son adquiridos/aprendidos en uso, a través de situaciones ejemplares o aplicaciones (que pueden ser de inclusión y también de exclusión) y no mediante definiciones, donde tales aplicaciones son indicativas (de manera imprecisa) de a qué otras aplicaciones cabe extender su uso;

(4) varios términos de género científicos son introducidos, y en su caso adquiridos/aprendidos, conjuntamente en una suerte de holismo epistémico-semántico local, consistente en que forman parte de ciertas generalizaciones que confieren a tales términos el carácter de proyectables; es decir, que tal holismo permite usar dichos términos para establecer (mediante condiciones más específicas que las dadas por aquellas generalizaciones) el

${ }^{23}$ Cfr. Kuhn 1991, p. 29 [v.c. p. 4]; 1993, p. 272 [v.c. p. 315 ].

${ }^{24}$ Kuhn (1991), p. 32 [v.c. p. 5].

${ }^{25}$ Cfr. Kuhn 1990, p. 302; 1993, pp. 292 [v.c. pp. 332-333]. 
comportamiento de las aplicaciones pretendidas y, por tanto, de sus entidades básicas; ${ }^{26}$

(5) las generalizaciones que convierten a los términos de género científicos en proyectables son unas pocas en una teoría, y proporcionan expectativas sobre las entidades básicas y complejas (sobre los referentes) a las(los) que conciernen tales términos; ${ }^{27}$

(6) tales generalizaciones son constitutivas, adquieren un rol estipulativo frente al rol empírico que tienen el resto de condiciones más específicas y en ese sentido los términos de género introducidos con dichas generalizaciones expresan conceptos que son constitutivos del objeto de conocimiento y lo categorizan;

(7) el status de tales generalizaciones con rol estipulativo no es el de proposiciones analíticas sino el de proposiciones sintéticas a priori, pero no se trata de una priori absoluto sino relativizado y de igual forma los conceptos de género introducidos mediante dichas generalizaciones son constitutivos de manera relativizada (no absoluta). ${ }^{28}$

Tras esta propuesta parece que se atisba la idea de que las generalizaciones que son sintético a priori relativizados no son otras que lo que hemos venido llamando "leyes fundamentales" de teorías maduras y complejas (de árboles teóricos), máxime a la luz del ejemplo paradigmático manejado por Kuhn: la segunda ley de la mecánica clásica, que es el ejemplo paradigmático de ley fundamental en la metateoría estructuralista. Yo efectivamente creo que las leyes fundamentales son sintéticos a priori relativizados, y que como tales juegan el papel que Kuhn les ha atribuido pero, si esto es así, podríamos encontrarnos en la situación de apostar por una nueva vuelta de tuerca para que la concepción estructuralista reciba el influjo del último Kuhn. Tomarse esto en serio conlleva apostar por los géneros en cuanto entidades abstractas - universales- como referencia de los términos científicos, y aceptar que con cada teoría algunos conceptos de género son constitutivos de (las partes de) la naturaleza sobre las que dicha teoría trata.

\footnotetext{
${ }^{26}$ Cfr. Kuhn 1993, pp. 273 y ss. [v.c. pp. 316 y ss.].

${ }^{27}$ Cfr. Kuhn 1993, p. 275 [v.c. p. 317].

${ }^{28}$ Cfr. Kuhn (1988), pp. 19 y ss.; (1990), pp. 306 y ss.; y (1993), pp. 290 y ss. [v.c. pp. 331 y ss.].
} 
Ello requiere de una ontosemántica en la que se tome en serio $^{29}$ a los géneros. Dicha ontosemántica no debe confundir la referencia de un término con su extensión conjuntista. ${ }^{30}$

Dejando a un lado ahora la cuestión ontosemántica, y retomando la equiparación de sintéticos a priori relativizados, habría que considerar si hay algún criterio delimitador de lo que es una ley fundamental. Kuhn no parece proporcionarlo explícitamente, y sólo da algún ejemplo paradigmático (además de exponer qué rol les corresponde). Tampoco las consideraciones estructuralistas previamente expuestas parecen llevarnos más lejos. No obstante, creo que Kuhn, y sobre todo la metateoría estructuralista, sí introducen elementos para establecer ese criterio delimitador.

\section{Los sintético a priori relativizados y ¿un criterio de ley fundamental?}

La idea de los a priori relativizados tiene su precedente al menos en un libro inicial de H. Reichenbach -a saber, Relativitätstheorie und Erkenntnis A priori [The Theory of Relativity and A Priori Knowledge] (cuyo original en alemán es de 1920)-, ${ }^{31}$ y recientemente en análisis de M. Friedman (que recupera la idea de Reichenbach), como el mismo Kuhn reconoce. En el caso de Reichenbach lo que éste hace es contraponer la idea de los a priori absolutos de Kant con una segunda acepción de a priori que es relativizado (supuestamente también debida a Kant). Conforme a esto, Reichenbach señala cómo ciertos postulados de la matemática y de la física son a priori en este sentido y no porque sean verdades necesarias, sino porque son "constitutivas del objeto" y cabe señalar que las de la física -y en general las propias de las ciencias empíricas- son sintéticos a priori. Con ello Reichenbach quiere dar cuenta del papel diferenciado de ciertas proposiciones, pero su análisis no va más allá de pretender salvar una noción de a priori que, pudiendo seguirse aplicando a ciertos principios presentes en la mecánica clásica, también se pueda aplicar a principios presentes en la mecánica relativista, aun

\footnotetext{
${ }^{29}$ En el sentido de ontología interna.

${ }^{30}$ Esta última es una línea que he venido defendiendo en algún trabajo, y que también ha venido desarrollando L. Villegas para los términos generales (en especial para los nombres comunes) Cfr. Falguera, 1992, 1997, 1998 y 2002; y Villegas 1997.

${ }^{31}$ Con posterioridad al texto de 1920 Reichenbach abandona la idea de los a priori, incluidos los relativizados.
} 
cuando no sean teorías compatibles. Reichenbach no nos proporciona un criterio delimitador de qué postulado es a priori en ese sentido constitutivo pero relativizado, y en concreto qué se requiere de un postulado de las teorías físicas para que ostente ese rol.

Friedman retoma la propuesta de Reichenbach en un afán de cuestionar la idea de una filosofía naturalizada que renuncie a todo tipo de a priori, aunque sin caer en la pretensión kantiana de establecer a priori absolutos. ${ }^{32}$ Con independencia de comentarios a la obra de Carnap y Quine -que incorpora en sus consideraciones sobre este asunto, y que ahora no vienen al caso-, Friedman llega a identificar tres niveles revolucionarios en las teorías mecánicas de Newton y de Einstein. Así, indica que uno de los niveles contiene, en cada una de dichas teorías, a priori matemáticos; a saber, el cálculo, para tratar con procesos que tienden al límite en el infinito en el caso de la teoría newtoniana (al que cabe añadir como a priori, no resultante de una revolución para la teoría newtoniana, a la geometría euclídea y a la cronometría clásica); y el cálculo de tensores y la teoría general multidimensional, en el caso de la einsteniana. Otro de los niveles contiene, en cada una de esas teorías, a priori sintéticos (físicos); a saber, nuevas concepciones de fuerza y cantidad de materia encapsuladas en sus tres leyes del movimiento, en la teoría newtoniana; y los principios de equivalencia de Einstein, que identifican efectos gravitacionales con efectos inerciales formalmente asociados con las leyes del movimiento de Newton, para la teoría general de la relatividad. El tercer nivel contiene proposiciones con contenido claramente empírico, como la ley de la gravitación universal, ${ }^{33}$ en la teoría newtoniana; y las ecuaciones de Einstein para el campo gravitacional, ${ }^{34}$ para la teoría general de la relatividad. El punto para Friedman estriba en mostrar que estos tres niveles están jerarquizados de manera tal que el último presupone, al menos metodológicamente, a los primeros, y a su vez el segundo al primero.

${ }^{32}$ Cfr. Friedman, 1997; 2000.

${ }^{33}$ La ley newtoniana de gravitación universal establece que entre dos cuerpos masivos hay una fuerza de atracción directamente proporcional al producto de sus masas e inversamente proporcional al cuadrado de la distancia que los separa.

${ }^{34}$ Las ecuaciones einstenianas para el campo gravitacional establecen cómo se modifica la curvatura del espacio-tiempo en presencia de materia y energía, y cómo se dirigen cuerpos afectados gravitacionalmente a lo largo de las rutas más rectas posibles o a lo largo de geodésicas. 
Pero tampoco Friedman proporciona un criterio de a priori, ni mucho menos de a priori sintético que podamos asimilar a nuestra noción de ley fundamental. Parece, pues, que se asume la existencia de 'a priori sintéticos y relativizados' sin que se disponga de un criterio de identidad para los mismos, lo que hace que la noción resulte filosóficamente, cuando menos, un tanto incómoda, o (si se prefiere) necesitada de elucidación.

En este punto quiero repescar los textos de Moulines sobre leyes fundamentales que ya he mencionado. Si volvemos el de 1991, Pluralidad y recursión, nos encontramos con el elemento insatisfactorio, a mi entender, de que presenta como leyes fundamentales a leyes como las más básicas de la mecánica de colisión, y prácticamente da a entender que en cada red teórica arbórea que podamos delimitar, con independencia de que forme parte de una más compleja, podemos establecer una ley fundamental. Obviamente este punto de vista supone una trivialización de la noción de ley fundamental que no hace parangón con los a priori sintéticos de Kuhn, Reichenbach y Friedman, y con su rol constitutivo.

Pero ya vimos que Moulines tiene un análisis previo sobre las leyes fundamentales. Se trata de aquél en el que denomina a las leyes fundamentales 'principios-guía'. Lo interesante de este texto es que toma ejemplos de más envergadura, como ya vimos. Uno de ellos es la segunda ley de Newton. El otro sería una ley general para la termodinámica reversible. De ambos señala que su cuasi-vacuidad se debe en última instancia a la forma lógica subyacente, que se caracteriza por estar presidida por cuantificadores existenciales de segundo orden (al menos), ${ }^{35}$ de manera tal que resultan claramente indeterminados o irrestrictos e irrefutables.

Centrémonos en el primer ejemplo, que sirve a Moulines para una elucidación más pormenorizada, dada su relevancia a la luz de la polémica histórica acerca de su carácter analítico (como mera definición) o sintético (como ley con contenido empírico). En relación con esta polémica Moulines tercia negando que el Segundo Principio de Newton sea una mera definición, pero añadiendo que:

${ }^{35} \mathrm{Cfr}$. Moulines 1987. No obstante el autor señala que no está claro que todos los principiosguía tenga que tener esa forma lógica ( $c f r$ ibídem, p. 89). 
... a pesar de que el Segundo Principio no puede tomarse como una definición, su estructura es tal que, en la terminología de Sneed, cualquier modelo parcial (no-teórico) puede ser «extendido» $\mathrm{o}$ «completado» trivialmente hasta transformarse en un modelo completo (teórico) de la mecánica, satisfaciendo por tanto el Segundo Principio. [...] En la reconstrucción de Sneed, el hecho de que el concepto de fuerza sólo sea discutible en términos del Segundo Principio y el hecho de que este principio sea empíricamente irrestricto son dos características, por así decir, «contingentes» de la mecánica clásica, casualidades históricas de la forma en la que se desarrolló la teoría y, por ende, dos casualidades desconectadas entre sí. (Moulines 1982, p. 96)

Frente a tal supuesta contingencia resultante del análisis sneediano, Moulines dice que la clave de la coincidencia estriba en que la cuantificación existencial de segundo orden alcanza a una variable funcional de fuerza (i.e., una variable para funciones de fuerza).

A la luz de tal comentario, y atendiendo a que también el ejemplo de ley fundamental o principio-guía de la termodinámica reversible tiene una forma lógica presidida por cuantificadores existenciales de orden superior, cabría pensar que tal forma lógica es peculiar de las leyes fundamentales; sin embargo, si bien cabe decir que ésta es una condición necesaria de las leyes fundamentales, ${ }^{36}$ no parece que sea una condición suficiente. Moulines mismo nos da un ejemplo de leyes que tienen esa forma lógica y que no cabe considerar leyes fundamentales de la teoría compleja de la que son parte (al menos a la luz de su desarrollo actual). Se trata de las leyes más básicas de la termodinámica de los sistemas simples (que es una sub-teoría de la termodinámica reversible). De nuevo estamos antes leyes que, según Moulines, están presididas por un cuantificador existencial que cuantifica sobre una variable de función, aunque en este caso es un único existencial y no una serie de ellos (como sucede en el Segundo Principio de Newton y en el principio básico de la termodinámica reversible). Dicho esto, Moulines no nos da más elementos para establecer qué es lo peculiar de una ley fundamental, aunque sí nos permite entender a éstas como proposiciones sintéticas a priori relativizadas; de hecho, habla del carácter

\footnotetext{
${ }^{36}$ Hay que reconocer que esto no pasa de ser una hipótesis que habría que constatar. Algunos interlocutores han señalado que tal vez no sea cierto que todas las leyes fundamentales tengan tal forma lógica. Tal vez tengan razón, pero en principio mi sospecha es que ese es un rasgo característico necesario de las leyes fundamentales.
} 
apriorístico del Segundo Principio de Newton y reconoce que dicho principio proporcionó, hablando en términos kantianos, las condiciones de posibilidad de la mecánica clásica. ${ }^{37}$

El criterio de ser sinóptico que Moulines contempla en Pluralidad y recursión, es decir, de contener todos los términos fundamentales (o característicos), ni siquiera sería una condición necesaria si atendemos al reconocimiento de Moulines de que habría excepciones constatadas como las leyes fundamentales de la mecánica relativista del continuo y de la electrodinámica (a la luz de la reconstrucción de Bartelborth).

Parece que se nos escurre la posibilidad de establecer elementos distintivos de las "leyes fundamentales"; sin embargo, creo que han estado presentes para la literatura estructuralista (y en cierta medida para Kuhn) sin que hayan sido explicitados. Desde mi punto de vista, la clave la encontramos en el criterio de teoricidad para distinguir los términos teóricos de los no-teóricos de una teoría dada $T$ (o los $T$-teóricos de los no $T$-teóricos). Hay una larga trayectoria en la elucidación de dicho criterio (en la versión pragmática) desde la propuesta intuitiva inicial de The Logical Structure of Mathematical Physics que ha tenido como artífices principales a los cuatro máximos exponentes de la metateoría estructuralista: Sneed, Stegmüller, Balzer y Moulines, y que encuentra su momento culminante en An Architectonic for Science. Atendiendo al criterio podemos plantear que una ley es fundamental para una teoría compleja madura (en un determinado momento) si y sólo si hay algún concepto de la teoría cuya determinación requiera siempre, en última instancia, que la ley en cuestión sea adecuada para al menos una aplicación, precisamente por ello una ley fundamental juega un papel constitutivo. ${ }^{38}$

Con esta propuesta, que liga el carácter de ley fundamental con el de criterio de teoricidad, no se exige que las leyes fundamentales incluyan todos los conceptos fundamentales o característicos de la teoría,

${ }^{37}$ Cfr. Moulines 1982, pp. 94 y 95.

${ }^{38}$ Jaramillo me ha hecho notar que Sellars identifica lo sintético a priori (relativizado) con "definiciones implícitas". La idea que hay detrás de la propuesta aquí defendida no es muy diferente, ya que el criterio de teoricidad viene a dar cuenta de por qué los términos teóricos de una teoría empírica son definidos implícitamente por su(s) ley(es) fundamenta(es). Obviamente, esto daría pie a que la categoría de a priori relativizado alcance también a postulados de teorías matemáticas (como asume Friedman); no obstante, yo dudaría en considerar éstos como "sintéticos" (a diferencia de lo que parece asumir Sellars). Cfr. Sellars 1963, cap. 10. 
aunque en muchos casos lo harán. Además, parte de su vacuidad la asegura esa cuantificación existencial. No entro en una consideración pormenorizada del criterio de teoricidad, pero debo recordar que la versión completa se establece para un holon teórico que supone árboles teóricos identificables.

La anterior propuesta, junto a la indicación de Moulines acerca de la forma lógica, nos permite ahora revisar las consideraciones de Friedman y mostrar que, por ejemplo, no son leyes fundamentales los tres principios newtonianos. Estos no están al mismo nivel, ya que (como nos recuerda Moulines) uno de ellos, el Primer Principio o de Inercia, se deriva trivialmente del Segundo, y el otro, el Tercer Principio, es más específico y por ello no válido para todas las aplicaciones de la teoría (por ejemplo para cargas en movimiento). Podemos decir, pues, que la concepción estructuralista proporciona un criterio para establecer por qué ciertas generalizaciones (a veces sólo una) de una teoría compleja son a priori sintéticos relativizados, como pretenden defender Kuhn y Friedman, y no simplemente indicar que hay tales cosas. El criterio permite discriminar entre las leyes más básicas de una teoría, cuál o cuáles son leyes fundamentales y cuál o cuáles son constitutivas de ciertas parcelas del mundo, es decir, mediante qué leyes se categoriza las parcelas con las que trata una determinada teoría.

\section{Referencias}

Allen, S. (comp.), 1988, Possible World in Humanities, Arts and Sciences, Walter de Gruyter, Berlín.

Asquith, P.D. y T. Nickles (comps.), 1983, PSA 1982, vol. 2, Philosophy of Science Association, East Lansing, Mich.

Balzer, W., C.U. Moulines, y J. Sneed, 1987, An Architectonic for Science. The Structuralist Program, Reidel, Dordrecht.

Boghossian, P. y C. Peacocke, 2000, New Essays on A Priori, Clarendon Press, Oxford.

Díez, J.A. y P. Lorenzano, 2002, Desarrollos actuales de la metateoría estructuralista: problemas y discusiones, Universidad Nacional de Quilmes, Quilmes.

Díez, J.A. y C.U. Moulines, 1997, Fundamentos de filosofía de la ciencia, Ariel, Barcelona.

Falguera, J.L., 1992, Problemas ontosemánticos de los términos científicos conforme a la concepción estructuralista, Tesis Doctoral (microficha), Servicios Públicos Universitarios de Santiago de Compostela, Santiago de Compostela. 
— 1997 , "A Basis for a Formal Semantics of Linguistic Formulations of Science”, en Ibarra y Mormann 1997, pp. 255-276.

—, 1998, "Inconmensurabilidad y Ontosemántica Representacional”, Teoría, vol. 13, no.1, pp. 161-185.

—, 2002 , "Ontosemántica de los términos científicos", en Díez y Lorenzano 2002, pp. 79-98.

Fine, A., M. Forbes y L. Wessels, 1991, PSA 1990, 2 Vol., Philosophy of Science Association, East Lansing, Mich.

Friedman M., 1997, "Philosophical Naturalism", Proceedings and Adresses of American Philosophical Association, vol. 71, no. 2, pp. 7-21.

—, 2000 , "Transcendental Philosophy and A Priori Knowledge: A Neo-Kantian Perspective”, en Boghossian y Peacocke 2000, pp. 367-383.

Ibarra, A. y T Mormann, 1997, Representations of Scientific Rationality. Contemporary Formal Philosophy of Science in Spain. Poznan Studies in the Philosophy of Sciences and the Humanities, vol. 61, Rodopi, Ámsterdam.

Krüger, L., L.J. Daston y M. Heidelberger (comps.) The Probabilistic Revolution, MIT Press, Cambridge.

Kuhn. T.S., 1962/1970, The Structure of Scientific Revolutions, University of Chicago Press, Chicago.

—, 1970, La Estructura de las Revoluciones Científicas, Fondo de Cultura Económica, México.

—, 1976, "Theory-Change as Structure-Change. Comments of the Sneed Formalism", en Erkenntnis, vol. 10, pp. 179-200.

— 1983 , "Commensurability, Comparability, Communicability", en Asquith y Nickles 1983, pp. 95-135.

—, 1987 , "What are Scientific Revolutions?”, en Krüger, Daston y Heidelberger 1987, pp. 7-22.

— 1988 , "Possible Worlds in History of Science", en Allen 1988, pp. 9-32-

_ 1990 , "Dubbing and Redubbing: The Vulnerability of Rigid Designation", en Savage 1990, pp. 298-318.

—, 1991, "The Road Since Structure", en Fine y Wessels 1991, pp. 2-13. Versión castellana: "El camino desde «La estructura»", Arbor, vol. 148, no. 583, pp. 27-46.

—, 1993, "Afterwords", en Horwich 1993, pp. 311-341.

Horwich, P., 1993, World Changes. Thomas Kuhn and the Nature of Science. The MIT Press, Cambridge.

Moulines, C.U., 1978, "Cuantificadores existenciales y principios-guía en las teorías físicas”, Crítica, vol. 10, pp. 59-88.

—, 1982, Exploraciones Metacientíficas, Alianza Editorial, Madrid.

—, 1991, Pluralidad y Recursión. Estudios Epistemológicos, Alianza Editorial, Madrid.

—, 2002, “¿Dónde se agazapa la pragmática en la representación estructural de las teorías?”, en Díez y Lorenzano 2002, pp. 99-115. 
Reichenbach, H., 1920, Relativitätstheorie und Erkenntnis A Priori, Springer, Berlín.

Savage, C.W. (comp.), 1990, Scientific Theories. (Minnesota Studies in the Philosophy of Science, vol. XIV), Minnesota University Press, Minneapolis.

Sellars, W., 1963, Science, Perception and Reality. Routledge and Kegan Paul, Londres.

Sneed, J. D., 1971, The Logical Structure of Mathematical Physics, Reidel, Dordrecht.

Squith, P. y T. Nickles, 1983, PSA 1992, vol. 2, East Leasing, Mich., pp. 669688. Versión castellana: "Conmensurabilidad, Comparabilidad y Comunicabilidad”, en Kuhn 1989.

Stegmüller, W., 1973, Theorienstrukturen und Theoriendynamik, Springer, Berlín. - 1976, "Accidental (Non-Substantial) Theory Change and Theory Dislogement", Erkenntnis, vol. 10, pp. 147-178.

—, 1978, "A Combined Approach to the Dynamics of Theories", Boston Studies in the Philosophy of Science, vol. 59, no. 136, pp. 151-186.

_, 1979, The Structuralist View of Theories, Springer, Berlín.

Villegas, L., 1997, "Common Names Are Not Concept-Words. The Introduction of Kinds in Fregean Ontology", Ruch Filozoficzny, vol. 54, no. 1, pp. $43-55$.

Recibido el 8 de septiembre de 2011 Aceptado el 30 de noviembre de 2011 